\title{
Meetings and Conferences
}

Visc Med 2018;34:402-404

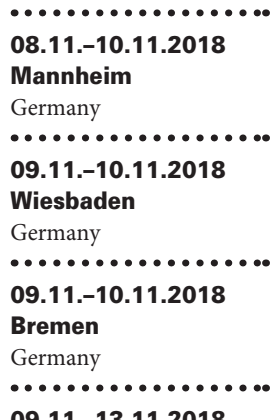

09.11.-13.11.2018

San Francisco, CA

USA

•..............

15.11.-16.11.2018

Paris

France

1.0.0.0.0.0.0.0.0.0.

15.11.-17.11.2018

Berlin

Germany

15.11.-17.11.2018

Mannheim

Germany

1..............

Innsbruck

Austria

•..............

15.11.-18.11.2018

Seoul

South Korea

16.11 .2018

Mainz

Germany

•................

16.11.-17.11.2018

Bad Kreuznach

Germany

..................

21.11.2018

Essen

Germany

-................

22.11.-23.11.2018

Frankfurt/M.

Germany

.................

27.11.-30.11.2018

Harrow

UK

•...............

30.11.-01.12.2018

Augsburg

Germany

(

05.12.-08.12.2018

Berlin

Germany

\section{DGVS-Seminar Onkologische Gastroenterologie - Modul 2}

12. Diabetes Herbsttagung der Deutschen Diabetes Gesellschaft (DAG) und 34. Jahrestagung der Deutschen Adipositas-Gesellschaft (DAG)

Herbsttagung 2018 der Norddeutschen Gesellschaft für Gastroenterologie

The Liver Meeting ${ }^{\circ} 2018$ - 69th Annual Meeting of the American Association for the Study of Liver Diseases (AASLD)

International Conference on Gastroenterology and Digestive Disorders

15. AlO-Herbstkongress - Update Medical Oncology

19. DGVS-Seminar Chronisch entzündliche Darmerkrankungen - Modul 2

17. Update Gastroenterologie-Stoffwechsel

Asian Pacific Digestive Week 2018 (APDW 2018)

Symposium: Viszeralmedizin 2018

33. Jahrestagung der Gastroenterologischen Arbeitsgemeinschaft Rheinland-Pfalz/Saarland

Best of Europe: State of the Art bei soliden Tumoren. Aktuelles vom Europäischen Krebskongress

10th Frankfurter Meeting

St. Mark's 16th Annual International Conference: Frontiers in Intestinal and Colorectal Disease

endo-update 2018

17. DGVS-Seminar Hepatologie - Modul 1
Information:

www.dgvs.de/fortbildung-aktuell/dgvs-veranstaltungen

\section{Information:}

www.herbsttagung-ddg.de

Information:

ndgg.org/index.php/kongresse.html

\section{Information:}

www.aasld.org/events-professional-development/

liver-meeting

Information:

gastroenterologists.alliedacademies.com/

\section{Information:}

www.aio-herbstkongress.de

\section{Information:}

www.dgvs.de/fortbildung-aktuell/dgvs-veranstaltungen

\section{Information:}

www.updategastro-stoffwechsel.at

\section{Information:}

www.apdw2018.org

\section{Information:}

www.viszeralmedizin2018.de

\section{Information:}

www.garps.de/

\section{Information:}

www.eickeler.org

\section{Information:}

www.frankfurter-meeting.de/

\section{Information:}

www.stmarksacademicinstitute.org.uk/courses/

\section{Information:}

www.endoupdate.de

\section{Information:}

www.dgvs.de/fortbildung-aktuell/dgvs-veranstaltungen

\section{KARGER}


¿... . . . . . . . .

Bangkok

Thailand

•...............

07.12.-08.12.2018

Amsterdam

The Netherlands

..................

15.12.2018

Vienna

Austria

17.01.-19.01.2019

San Francisco, CA

USA

[... . . . . . . . . .

Berlin

Germany

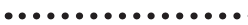

07.02.-09.02.2019

Düsseldorf

Germany

... . . . . . . . . . .

Göttingen

Germany

.................

07.02.-09.02.2019

Hanover

Germany

09.02.2019

Munich

Germany

................

22.02.-23.02.2019

Potsdam

Germany

.................

04.03.-06.03.2019

Berlin

Germany

......................

06.03.-09.03.2019

Berlin

Germany

................

06.03.-09.03.2019

Copenhagen

Denmark

.................

28.03.-30.03.2019

Stuttgart

Germany

•..............

04.04.-06.04.2019

Prague

Czech Republic

.................

10.04.-14.04.2019

Vienna

Austria

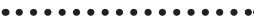

04.05.-07.05.2019

Wiesbaden

Germany

-....................

10.05.-11.05.2019

Berlin

Germany

•................

10.05.-11.05.2019

Halle

Germany
Gastro 2018: WGO-GAT International Conference

International Viral Hepatitis Elimination Meeting (IVHEM)

Gastro-Highlights 2018

Gastrointestinal Cancers Symposium

13. Jahrestagung der Gesellschaft für Gastroenterologie und Hepatologie in Berlin und Brandenburg

21st Düsseldorf International Endoscopy Symposium

39. Jahrestagung des Deutschen Pankreasclubs (DPC)

Jahrestagung 2019 der Norddeutschen Gesellschaft für Gastroenterologie

11. Jahreskongress des Tumorzentrums München (TZM Essentials 2019)

6. Brandenburger Krebskongress

20th World Congress on Gastroenterology

17. DGVS-Seminar Hepatologie - Modul 2

Inflammatory Bowel Diseases: 14th Congress of ECCO

49. Kongress der Deutschen Gesellschaft für Endoskopie und Bildgebende Verfahren e.V. (DGE-BV)

\section{ESGE Days 2019}

The International Liver Congress ${ }^{\mathrm{TM}} 2019$

125. Jahrestagung der Deutschen Gesellschaft für Innere Medizin

Endoskopie Live 2019

2. Gemeinsame Jahrestagung der Mitteldeutschen Chirurgenvereinigung und der Mitteldeutschen Gesellschaft für Gastroenterologie
Information:

www.gastro2018bangkok.com

Information:

www.virology-education.com/event/upcoming/

5th-ivhem-2018/

Information:

www.gastrohighlights.org/

Information:

gicasym.org

\section{Information:}

www.gghbb.de

\section{Information:}

www.endo-duesseldorf.com

\section{Information:}

www.dpc-kongress.de/

\section{Information:}

ndgg.org/index.php/kongresse.html

\section{Information:}

www.tzm-essentials.de

\section{Information:}

www.mcall-gmbh.de/bb-krebskongress/

\section{Information:}

gastro.insightconferences.com

\section{Information:}

www.dgvs.de/fortbildung-aktuell/dgvs-veranstaltungen

\section{Information:}

www.ecco-ibd.eu/ecco19

\section{Information:}

www.dge-bv.de/

Information:

esgedays.org

\section{Information:}

www.easl.eu/discover/events/detail/2019/

the-international-liver-congress-2019

.............................

Information:

dgim2019.de

\section{Information:}

www.endoskopie-live-berlin.de

Information:

www.mgfg.de 
18.05.-21.05.2019

San Diego, CA

USA

24.05.-25.05.2019

Ludwigshafen

Germany

.................

02.06.-05.06.2019

Amsterdam

The Netherlands

.................

05.06.-07.06.2019

Basel

Switzerland

..................

06.06.-07.06.2019

Essen

Germany

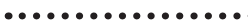

13.06.-15.06.2019

Innsbruck

Austria

15.06.2019

Wiesbaden

Germany

................

27.06.-28.06.2019

Passau

Germany

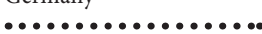

03.07.-06.07.2019

Barcelona

Spain

.................

08.07.-09.07.2019

Zurich

Switzerland

..................

12.09.-13.09.2019

Interlaken

Switzerland

.................

19.09.-20.09.2019

St. Gallen

Switzerland

................

19.09.-21.09.2019

Salzburg

Austria

.................

21.09.-24.09.2019

Istanbul

Turkey

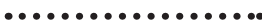

27.09.-01.10.2019

Barcelona

Spain

..................

02.10.-05.10.2019

Wiesbaden

Germany

................

11.10.-14.10.2019

Berlin

Germany

.................

01.11.-02.11.2019

Berlin

Germany

.................

21.11.-24.11.2019

Kobe

Japan
Digestive Disease Week $^{\circledR}$

7. Ludwigshafener GastroTage 2019

13th Congress of the European-African Hepato-Pancreato-Biliary Association (E-AHPBA)

4. Frühjahrskongress der Schweizerischen Gesellschaft für Allgemeine Innere Medizin (SGAIM)

Viszeralmedizin NRW 2019

52. Jahrestagung \& 30 . Fortbildungskurs der ÖGGH

GI-Oncology 2019: 15. Interdisziplinäres Update

47. Jahrestagung der Gesellschaft für Gastroenterologie in Bayern e.V.

World Congress on Gastrointestinal Cancer

14th Euro-Global Gastroenterology Conference

Jahreskongress der Schweizerischen Gesellschaft für Gastroenterologie

Herbstkongress der Schweizerischen Gesellschaft für Allgemeine Innere Medizin

50. Jahrestagung der Österreichischen Gesellschaft für Innere Medizin

World Congress of Gastroenterology 2019

ESMO 2019 Congress

Viszeralmedizin 2019: 74. Jahrestagung der DGVS und 13. Herbsttagung der DGAV

Jahrestagung der Deutschen, Österreichischen und Schweizerischen Gesellschaften für Hämatologie und Medizinische Onkologie

Endo Club Nord 2019

JDDW 2019 - Japan Digestive Disease Week 2019
Information:

www.ddw.org/home

Information:

www.gastrotagelu.de

Information:

www.e-ahpba2019.com

Information:

congress.sgaim.ch/de/fruehjahrskongress.html

\section{Information:}

viszeralmedizin-nrw.de

\section{Information:}

www.oeggh.at/veranstaltungen

Information:

www.gi-oncology.de

\section{Information:}

www.gfgb.org/tagung-2019.php

\section{Information:}

www.worldgicancer.com/

\section{Information:}

europegastroenterology.gastroconferences.com

\section{Information:}

www.sggssg.ch/veranstaltungen-sgg/jahreskongress

Information :

congress.sgaim.ch/de/herbstkongress.html

Information :

www.oegim.at/aktuell/jahrestagung.html

\section{Information:}

www.worldgastroenterology.org/meetings-and-events/

meetings-and-events-calendar

•.................................

Information:

www.esmo.org/Conferences/ESMO-2019-Congress

\section{Information:}

www.viszeralmedizin.com/zukuenftige-kongresse/

viszeralmedizin-2019

Information:

www.dgho.de/veranstaltungen/jahrestagung/zukuenftige-

jahrestagungen

Information:

www.endoclubnord.de

Information:

www.jddw.jp/jddw2019/en/index.html 